\title{
Positive solutions for singular higher-order semipositone fractional differential equations with conjugate type integral conditions
}

\author{
Qiuyan Zhong ${ }^{\mathrm{a}}$, Xingqiu Zhang ${ }^{\mathrm{b}, *}$, Zhuyan Shao ${ }^{\mathrm{b}}$ \\ ${ }^{a}$ Center for Information Technology, Jining Medical University, Jining, 272067, P. R. China. \\ ${ }^{b}$ School of Medical Information Engineering, Jining Medical University, Rizhao, 276826, P. R. China.
}

Communicated by Y. H. Yao

\begin{abstract}
In this article, height functions on different bounded sets of the nonlinear term and their integrations are considered to obtain the existence of positive solutions for a class of semipositone higher-order fractional differential equations with nonlocal conjugate type integral conditions. The singularities of the nonlinearity are related to both the time and the space variables. (C)2017 All rights reserved.
\end{abstract}

Keywords: Fractional differential equations, conjugate type integral conditions, positive solution, semipositone, singularity. 2010 MSC: 26A33, 34B15, 34B16.

\section{Introduction}

The purpose of this article is to consider the existence of positive solutions for the following fractional differential equation

$$
\mathrm{D}_{0+}^{\alpha} \mathrm{u}(\mathrm{t})+\mathrm{f}(\mathrm{t}, \mathrm{u}(\mathrm{t}))=0, \quad 0<\mathrm{t}<1,
$$

with nonlocal conjugate type integral conditions

$$
u(0)=u^{\prime}(0)=\cdots=u^{(n-2)}(0)=0, \quad D_{0+}^{\beta} u(1)=\lambda \int_{0}^{\eta} h(t) D_{0+}^{\beta} u(t) d t,
$$

where $D_{0+}^{\alpha}$ is the standard Riemann-Liouville derivative, $h \in L^{1}[0,1]$ is nonnegative and may be singular at $t=0$ and $t=1, n-1<\alpha \leqslant n, n \geqslant 3, \beta \geqslant 1, \alpha-\beta-1>0,0<\eta \leqslant 1,0 \leqslant \lambda \int_{0}^{\eta} h(t) t^{\alpha-\beta-1} d t<1$.

The concept of fractional derivative has a long history over 300 years. At the early stage of its emergence, it was considered only by very few mathematicians in pure mathematical field for the lack of practical applications. In the last decades, more and more researchers have realized that fractional calculus is one of the best tools to describe long-memory process. At the same time, scholars have recognized

\footnotetext{
*Corresponding author

Email addresses: zhqy197308@163.com (Qiuyan Zhong), zhxq197508@163.com (Xingqiu Zhang), 317096018@qq.com (Zhuyan Shao)

doi:10.22436/jnsa.010.09.37
} 
that it can be applied to almost every field of science, engineering and mechanics among which fractional calculus has made a profound impact including viscoelasticity and rheology, electrochemistry, biophysics and bioengineering, signal and image processing, control theory, mechanics, mechatronics, economics and so on (see [30]). It is also helpful to see the books and monographs [6, 25, 29, 31, 45] for further understanding how the fractional calculus works in the field of engineering, science, economics and finance, pure and applied mathematics communities.

The investigation on $(p, n-p)$ conjugate boundary value problems

$$
\left\{\begin{array}{l}
(-1)^{(n-k)} u^{(n)}(t)=\phi(t) f(t, u(t)), \quad 0<t<1 \\
u^{(i)}(0)=0, \quad 0 \leqslant i \leqslant p-1 \\
u^{(j)}(1)=0, \quad p \leqslant j \leqslant n-1
\end{array}\right.
$$

can be back to some well-known existing papers [1-3, 9-13, 24, 27, 32] published a couple of decades ago. When $n=2$, conjugate boundary value problems can be widely applied to describe important phenomena which arise in boundary layer theory, reaction-diffusion theory and in non-Newtonian fluid theory. When $n=4$, it can describe the deflection of an elastic beam rigidly fixed at both ends, or one end is simply supported whereas the other end is clamped by sliding clamps. For singular boundary value problems, researchers paid more attention on the existence of positive solutions. By means of classic Agarwal-O'Regan method, existence of single, twin, and multiple results are obtained in [1-3]. The characteristic of Agarwal-O'Regan method lies in two aspects. One is that $\phi(t)$ may be singular at $t=0,1$ and $f(t, u)$ may be singular at $u=0$, respectively. The other is there is no requirements on the existence of lower and upper solutions. For instance, under the following assumptions which can be looked as classic and traditional conditions of Agarwal-O'Regan method, [3] gave the existence of two solutions for $(p, n-p)$ conjugate boundary value problems.

$\left(\mathrm{p}_{1}\right) \phi \in \mathrm{C}(0,1)$ with $\phi>0$ on $(0,1)$;

$\left(\mathrm{p}_{2}\right) \mathrm{f}:[0,1] \times(0, \infty) \rightarrow(0, \infty)$ is continuous;

$\left(p_{3}\right) f(t, u) \leqslant g(u)+h(u)$ on $[0,1] \times(0, \infty)$ with $g>0$ continuous and nonincreasing on $(0, \infty), h \geqslant 0$ continuous on $[0, \infty)$ and $\frac{h}{g}$ nondecreasing on $(0, \infty)$;

$\left(p_{4}\right)$ there exists $K_{0}$ with $g(a b) \leqslant K_{0} g(a) g(b)$ for all $a \geqslant 0, b \geqslant 0$;

$\left(\mathrm{p}_{5}\right) \int_{0}^{1} \phi(\mathrm{s}) \mathrm{g}\left(\mathrm{s}^{\mathrm{p}}\right) \mathrm{d} \mathrm{s}<\infty$;

$\left(p_{6}\right)$ for each constant $H>0$ there exists $\psi_{H}$ continuous on $[0,1]$ and positive on $(0,1)$ such that $f(t, u) \geqslant$ $\psi_{\mathrm{H}}(\mathrm{t})$ on $[0,1] \times(0, \mathrm{H}]$ and

$$
\exists r>0 \text { with } \frac{r}{g(r)+h(r)}>c_{0} K_{0},
$$

here $c_{0}=\sup _{t \in[0,1]} \int_{0}^{1}(-1)^{n-p} G(t, s) \phi(s) g\left(s^{p}\right) d s ;$

$\left(p_{7}\right)$ there exists $a \in\left(0, \frac{1}{2}\right)$ (choose and fix it) and $\tau \in C[a, 1-a]$ with $\tau>0$ on $[a, 1-a]$ and with $\phi(t) f(t, u) \geqslant \tau(t)[g(u)+h(u)]$ on $[a, 1-a] \times(0, \infty)$ and there exists $R>r$ with

$$
\frac{\operatorname{Rg}\left(a^{p} R\right)}{g(R) g\left(a^{p} R\right)+g(R) h\left(a^{p} R\right)} \leqslant \int_{a}^{1-a}(-1)^{n-p} G(\sigma, s) \tau(s) d s,
$$

here $0 \leqslant \sigma \leqslant 1$ is such that

$$
\int_{a}^{1-a}(-1)^{n-p} G(\sigma, s) \tau(s) d s=\sup _{t \in[0,1]} \int_{a}^{1-a}(-1)^{n-p} G(t, s) \tau(s) d s .
$$

On the other hand, due to the deep background in various fields of applied mathematics and physics such as heat conduction, chemical engineering, underground water flow, thermo-elasticity, and plasma physics, there has been a great increase in investigating differential equations under nonlocal conditions, (see $[4,7,8,14,15,17-23,26,28,33-35,38-44]$ to name a few). Recently, Webb [35] and Hao et al. [20] 
considered a kind of $n$ th-order differential equations subject to the following conjugate type boundary value conditions

$$
u(0)=u^{\prime}(0)=\cdots=u^{(n-2)}(0)=0, u(1)=\int_{0}^{1} u(s) d A(s),
$$

which covers multi-point boundary conditions and integral conditions in a unified framework. They obtained the existence of positive solutions for the BVPs under some conditions concerning to the principal eigenvalue of the corresponding linear operator when the nonlinearity $f$ either satisfies Carathéodory conditions or permits singularity with respect to both the time and the space variables. By means of the fixed point theory, Wang et al. [33] investigated the existence and multiplicity of positive solution for fractional differential equations BVP (1.1) and (A). Very recently, when $f$ is continuous, Cabada et al. [8] considered the existence of positive solutions for the following fractional differential equations with a parameter $\lambda$ in integral boundary value conditions

$$
\mathfrak{u}(0)=\mathfrak{u}^{\prime \prime}(0)=0, \mathfrak{u}(1)=\lambda \int_{0}^{1} u(s) d s,
$$

where $0<\lambda<2,{ }^{C} D^{\alpha}$ is the Caputo fractional derivative. Motivated by [8], the authors [34, 39, 44] investigated higher order fractional differential equation (1.1) under more general integral boundary conditions

$$
\begin{aligned}
& u(0)=u^{\prime}(0)=u^{\prime \prime}(0)=0, u(1)=\lambda \int_{0}^{\eta} u(s) d s, \\
& u(0)=u^{\prime}(0)=\cdots=u^{(n-2)}(0)=0, \quad u^{(i)}(1)=\lambda \int_{0}^{\eta} u(t) d t, \\
& u(0)=u^{\prime}(0)=\cdots=u^{(n-2)}(0)=0, \quad u^{(i)}(1)=\lambda \int_{0}^{\eta} h(t) u(t) d t,
\end{aligned}
$$

where $i$ is a nonnegative integer, $\eta \in(0,1]$, and $\lambda$ is a positive parameter.

The purpose of this paper is to investigate the existence of positive solutions for fractional differential equations BVP (1.1)-(1.2) under two cases, that is to say, when $f(t, u)$ is singular with respect to time variable and singular with respect to both the time and the space variables. It should be pointed out that the nonlinearity may change sign which makes the problem considered in this article be so-called semipositone problems. Compared to the literature, this paper has several new features. Firstly, the nonlinearity $f$ may be unbounded below. Especially, it is worth mentioning that the nonlinearity permits singularities with both the time and the space variables. Secondly, compared to $[8,34,39,44]$, an arbitrary order derivative, a Lebesgue integrable function $h$ and two parameters $\lambda$ and $\eta$ are involved in the integral boundary conditions which makes the BVP (1.1)-(1.2) has a more general form. Thirdly, and it is most important that the conditions imposed on the nonlinearity and the method of the proof are different from the existing results $[1-3,27]$. More precisely, height functions of the nonlinear term on different bounded sets for each case are introduced and the integrations of these height functions are considered to guarantee the existence of positive solutions. Conditions used in this paper are easy to be checked and they can also be easily employed to obtain the multiplicity results of positive solutions for other boundary value problems.

The idea of this paper may trace to Yao [36, 37] and our work [38]. As far as we know, there are few papers to consider fractional semipositone integral BVPs, especially for BVPs with singularities on space variable. Our proof is based on a special cone constructed according to the properties of the corresponding Green's function. To gain this goal, we carried out a detailed discussion on the properties of Green's function.

\section{Preliminaries and several lemmas}

Let $E=C[0,1],\|u\|=\max _{0 \leqslant t \leqslant 1}|u(t)|$, then $(E,\|\cdot\|)$ is a Banach space. In this article, we still need another Banach space $\mathrm{L}^{1}(0,1)$ endowed with the norm $\|\mathrm{u}\|_{1}=\int_{0}^{1}|\mathrm{u}(\mathrm{t})| \mathrm{dt}$. 
Definition 2.1 ([25]). The Riemann-Liouville fractional integral of order $\alpha>0$ of a function $y:(0, \infty) \rightarrow R$ is given by

$$
\mathrm{I}_{0+}^{\alpha} \mathrm{y}(\mathrm{t})=\frac{1}{\Gamma(\alpha)} \int_{0}^{\mathrm{t}}(\mathrm{t}-\mathrm{s})^{\alpha-1} \mathrm{y}(\mathrm{s}) \mathrm{d} s
$$

provided the right-hand side is pointwise defined on $(0, \infty)$.

Definition 2.2 ([25]). The Riemann-Liouville fractional derivative of order $\alpha>0$ of a continuous function $y:(0, \infty) \rightarrow R$ is given by

$$
D_{0+}^{\alpha} y(t)=\frac{1}{\Gamma(n-\alpha)}\left(\frac{d}{d t}\right)^{n} \int_{0}^{t} \frac{y(s)}{(t-s)^{\alpha-n+1}} d s,
$$

where $n=[\alpha]+1,[\alpha]$ denotes the integer part of the number $\alpha$, provided that the right-hand side is pointwise defined on $(0, \infty)$.

Lemma 2.3 ([5]). Let $\alpha>0$. If we assume $u \in C(0,1) \cap \mathrm{L}(0,1)$, then the fractional differential equation

$$
\mathrm{D}_{0+}^{\alpha} \mathrm{u}(\mathrm{t})=0,
$$

has $\mathrm{u}(\mathrm{t})=\mathrm{C}_{1} \mathrm{t}^{\alpha-1}+\mathrm{C}_{2} \mathrm{t}^{\alpha-2}+\cdots+\mathrm{C}_{\mathrm{N}} \mathrm{t}^{\alpha-\mathrm{N}}, \mathrm{C}_{\mathrm{i}} \in \mathrm{R}, \mathrm{i}=1,2, \ldots, \mathrm{N}$, as unique solutions, where $\mathrm{N}$ is the smallest integer greater than or equal to $\alpha$.

Lemma 2.4 ([5]). Assume that $u \in C(0,1) \cap \mathrm{L}(0,1)$ with a fractional derivative of order $\alpha>0$ that belongs to $\mathrm{C}(0,1) \cap \mathrm{L}(0,1)$. Then

$$
I_{0+}^{\alpha} D_{0+}^{\alpha} u(t)=u(t)+C_{1} t^{\alpha-1}+C_{2} t^{\alpha-2}+\cdots+C_{N} t^{\alpha-N}
$$

for some $C_{i} \in R, i=1,2, \ldots, N$, where $N$ is the smallest integer greater than or equal to $\alpha$.

Lemma 2.5. Assume that $\lambda \int_{0}^{\eta} h(t) t^{\alpha-\beta-1} \mathrm{dt} \neq 1$. Then for any $y \in \mathrm{L}^{1}(0,1)$, the unique solution of the boundary value problems

$$
\left\{\begin{array}{l}
D_{0+}^{\alpha} u(t)+y(t)=0,0<t<1, \\
u(0)=u^{\prime}(0)=\cdots=u^{(n-2)}(0)=0, D_{0+}^{\beta}(1)=\lambda \int_{0}^{\eta} h(t) D_{0+}^{\beta} u(t) d t,
\end{array}\right.
$$

can be expressed in the form

$$
u(t)=\int_{0}^{1} G(t, s) y(s) d s, t \in[0,1]
$$

where

$$
\begin{aligned}
& \mathrm{G}(\mathrm{t}, \mathrm{s})=\mathrm{G}_{1}(\mathrm{t}, \mathrm{s})+\mathrm{G}_{2}(\mathrm{t}, \mathrm{s}), \\
& G_{1}(t, s)= \begin{cases}\frac{t^{\alpha-1}(1-s)^{\alpha-\beta-1}-(t-s)^{\alpha-1}}{\Gamma(\alpha)}, & 0 \leqslant s \leqslant t \leqslant 1, \\
\frac{t^{\alpha-1}(1-s)^{\alpha-\beta-1}}{\Gamma(\alpha)}, & 0 \leqslant t \leqslant s \leqslant 1,\end{cases} \\
& G_{2}(t, s)=\frac{\lambda t^{\alpha-1}}{1-\lambda \int_{0}^{\eta} h(t) t^{\alpha-\beta-1} d t} \int_{0}^{\eta} h(t) H(t, s) d t \text {, } \\
& H(t, s)= \begin{cases}\frac{t^{\alpha-\beta-1}(1-s)^{\alpha-\beta-1}-(t-s)^{\alpha-\beta-1}}{\Gamma(\alpha)}, & 0 \leqslant s \leqslant t \leqslant 1, \\
\frac{t^{\alpha-\beta-1}(1-s)^{\alpha-\beta-1}}{\Gamma(\alpha)}, & 0 \leqslant t \leqslant s \leqslant 1,\end{cases}
\end{aligned}
$$

here, $\mathrm{G}(\mathrm{t}, \mathrm{s})$ is called the Green function of $B V P(2.1)$. Obviously, $\mathrm{G}(\mathrm{t}, \mathrm{s})$ is continuous on $[0,1] \times[0,1]$. 
Proof. First, (2.1) can be transformed to an equivalent integral equation by Lemma 2.4,

$$
\begin{aligned}
u(t) & =-I_{0+}^{\alpha} y(t)+C_{1} t^{\alpha-1}+C_{2} t^{\alpha-2}+\cdots+C_{n} t^{\alpha-n} \\
& =-\frac{1}{\Gamma(\alpha)} \int_{0}^{t}(t-s)^{\alpha-1} y(s) d s+C_{1} t^{\alpha-1}+C_{2} t^{\alpha-2}+\cdots+C_{n} t^{\alpha-n}
\end{aligned}
$$

for some $C_{1}, C_{2}, \cdots, C_{n} \in R$. Combining with the boundary value conditions that $u(0)=u^{\prime}(0)=\cdots=$ $u^{(n-2)}(0)=0$, one gets that $C_{2}=C_{3}=\cdots=C_{n}=0$. Then, we have

$$
u(t)=-\int_{0}^{t} \frac{(t-s)^{\alpha-1}}{\Gamma(\alpha)} y(s) d s+C_{1} t^{\alpha-1}
$$

By Definition 2.2, we get

$$
D_{0+}^{\beta} u(t)=-\int_{0}^{t} \frac{(t-s)^{\alpha-\beta-1}}{\Gamma(\alpha-\beta)} y(s) d s+C_{1} \frac{\Gamma(\alpha)}{\Gamma(\alpha-\beta)} t^{\alpha-\beta-1}
$$

Thus, we have

$$
\begin{aligned}
D_{0+}^{\beta} u(1)= & -\int_{0}^{1} \frac{(1-s)^{\alpha-\beta-1}}{\Gamma(\alpha-\beta)} y(s) d s+C_{1} \frac{\Gamma(\alpha)}{\Gamma(\alpha-\beta)} \\
\lambda \int_{0}^{\eta} h(t) D_{0+}^{\beta} u(t) d t= & \lambda\left[\int_{0}^{\eta} h(t)\left(-\frac{1}{\Gamma(\alpha-\beta)}\right) \int_{0}^{t}(t-s)^{\alpha-\beta-1} y(s) d s d t\right. \\
& \left.+C_{1} \frac{\Gamma(\alpha)}{\Gamma(\alpha-\beta)} \int_{0}^{\eta} h(t) t^{\alpha-\beta-1} d t\right]
\end{aligned}
$$

By (2.4) and (2.5), we get that

$$
\begin{aligned}
C_{1} \frac{\Gamma(\alpha)}{\Gamma(\alpha-\beta)}\left(1-\lambda \int_{0}^{\eta} h(t) t^{\alpha-\beta-1} d t\right)= & \frac{1}{\Gamma(\alpha-\beta)}\left(\int_{0}^{1}(1-s)^{\alpha-\beta-1} y(s) d s\right. \\
& \left.-\lambda \int_{0}^{\eta} h(t) \int_{0}^{t}(t-s)^{\alpha-\beta-1} y(s) d s d t\right) .
\end{aligned}
$$

As a result,

$$
C_{1}=\frac{1}{\Gamma(\alpha)\left(1-\lambda \int_{0}^{\eta} h(t) t^{\alpha-\beta-1} d t\right)}\left(\int_{0}^{1}(1-s)^{\alpha-\beta-1} y(s) d s-\lambda \int_{0}^{\eta} h(t) \int_{0}^{t}(t-s)^{\alpha-\beta-1} y(s) d s d t\right) .
$$

Thus,

$$
\begin{aligned}
u(t)= & -\frac{1}{\Gamma(\alpha)} \int_{0}^{t}(t-s)^{\alpha-1} y(s) d s+\frac{t^{\alpha-1}}{\Gamma(\alpha)\left(1-\lambda \int_{0}^{\eta} h(t) t^{\alpha-\beta-1} d t\right)} \\
& \cdot\left(\int_{0}^{1}(1-s)^{\alpha-\beta-1} y(s) d s-\lambda \int_{0}^{\eta} h(t) \int_{0}^{t}(t-s)^{\alpha-\beta-1} y(s) d s d t\right) \\
= & -\frac{1}{\Gamma(\alpha)} \int_{0}^{t}(t-s)^{\alpha-1} y(s) d s+\frac{\left(1-\lambda \int_{0}^{\eta} h(t) t^{\alpha-\beta-1} d t+\lambda \int_{0}^{\eta} h(t) t^{\alpha-\beta-1} d t\right)}{\Gamma(\alpha)\left(1-\lambda \int_{0}^{\eta} h(t) t t^{\alpha-\beta-1} d t\right)} t^{\alpha-1} \\
& \cdot \int_{0}^{1}(1-s)^{\alpha-\beta-1} y(s) d s-\frac{\lambda t^{\alpha-1}}{\Gamma(\alpha)\left(1-\lambda \int_{0}^{\eta} h(t) t^{\alpha-\beta-1} d t\right)}
\end{aligned}
$$




$$
\begin{aligned}
& \cdot \int_{0}^{\eta} h(t) \int_{0}^{t}(t-s)^{\alpha-\beta-1} y(s) d s d t \\
= & -\frac{1}{\Gamma(\alpha)} \int_{0}^{t}(t-s)^{\alpha-1} y(s) d s+\frac{1}{\Gamma(\alpha)} \int_{0}^{1} t^{\alpha-1}(1-s)^{\alpha-\beta-1} y(s) d s \\
& +\frac{\lambda}{\Gamma(\alpha)} \frac{\int_{0}^{1}(1-s)^{\alpha-\beta-1} y(s) d s}{1-\lambda \int_{0}^{\eta} h(t) t^{\alpha-\beta-1} d t} t^{\alpha-1} \cdot \int_{0}^{\eta} h(t) t^{\alpha-\beta-1} d t \\
& -\frac{\lambda}{\Gamma(\alpha)} \frac{t^{\alpha-1}}{1-\lambda \int_{0}^{\eta} h(t) t^{\alpha-\beta-1} d t} \int_{0}^{\eta} h(t) \int_{0}^{t}(t-s)^{\alpha-\beta-1} y(s) d s d t \\
= & \int_{0}^{1} G_{1}(t, s) y(s) d s+\frac{\lambda}{\Gamma(\alpha)} \frac{t^{\alpha-1}}{1-\lambda \int_{0}^{\eta} h(t) t^{\alpha-\beta-1} d t} \\
& \cdot\left[\int_{0}^{\eta}\left(\int_{0}^{1}(1-s)^{\alpha-\beta-1} y(s) h(t) t^{\alpha-\beta-1} d s\right) d t-\int_{0}^{\eta} h(t) \int_{0}^{t}(t-s)^{\alpha-\beta-1} y(s) d s d t\right] \\
= & \int_{0}^{1} G_{1}(t, s) y(s) d s+\frac{\lambda}{\Gamma(\alpha)} \frac{t^{\alpha-1}}{1-\lambda \int_{0}^{\eta} h(t) t^{\alpha-\beta-1} d t} \\
& \cdot\left[\int_{0}^{\eta} h(t) \int_{0}^{t}\left((1-s)^{\alpha-\beta-1} t^{\alpha-\beta-1}-(t-s)^{\alpha-\beta-1}\right) y(s) d s d t\right. \\
& \left.+\int_{0}^{\eta} h(t) \int_{t}^{1}(1-s)^{\alpha-\beta-1} t^{\alpha-\beta-1} y(s) d s d t\right] \\
= & \int_{0}^{1} G_{1}(t, s) y(s) d s+\frac{\lambda t^{\alpha-1}}{1-\lambda \int_{0}^{\eta} h(t) t^{\alpha-\beta-1} d t} \cdot \int_{0}^{\eta} h(t) \int_{0}^{1} H(t, s) y(s) d s d t \\
= & \int_{0}^{1} G_{1}(t, s) y(s) d s+\int_{0}^{1} \frac{\lambda t^{\alpha-1}}{1-\lambda \int_{0}^{\eta} h(t) t^{\alpha-\beta-1} d t} \cdot \int_{0}^{\eta} h(t) H(t, s) d t y(s) d s \\
= & \int_{0}^{1}\left(G_{1}(t, s)+G_{2}(t, s)\right) y(s) d s . \\
& \\
&
\end{aligned}
$$

Lemma 2.6. The functions $\mathrm{G}_{1}(\mathrm{t}, \mathrm{s})$ and $\mathrm{G}(\mathrm{t}, \mathrm{s})$ given by (2.3) and (2.2), respectively, have the following properties:

$\left(a_{1}\right) G_{1}(t, s) \geqslant \frac{1}{\Gamma(\alpha)} t^{\alpha-1} s(1-s)^{\alpha-\beta-1}, \forall t, s \in[0,1] ;$

$\left(a_{2}\right) G_{1}(t, s) \leqslant \frac{1}{\Gamma(\alpha)}(\alpha-1) s(1-s)^{\alpha-\beta-1}, \forall t, s \in[0,1]$;

$\left(a_{3}\right) \mathrm{G}(\mathrm{t}, \mathrm{s}) \leqslant J(s), J(s)=\frac{1}{\Gamma(\alpha)}(\alpha-1) s(1-s)^{\alpha-\beta-1}+\frac{\lambda}{1-\lambda \int_{0}^{\eta} \mathrm{h}(\mathrm{t}) \mathrm{t}^{\alpha-\beta-1} \mathrm{dt}} \int_{0}^{\eta} \mathrm{h}(\mathrm{t}) \mathrm{H}(\mathrm{t}, \mathrm{s}) \mathrm{dt}, \forall \mathrm{t}, \mathrm{s} \in[0,1]$;

$\left(a_{4}\right) \frac{1}{(\alpha-1)} t^{\alpha-1} J(s) \leqslant G(t, s) \leqslant \frac{1}{\Gamma(\alpha)} t^{\alpha-1}(1-s)^{\alpha-\beta-1}\left[(\alpha-1)+\frac{\lambda}{1-\lambda \int_{0}^{\eta} h(t) t^{\alpha-\beta-1} d t} \int_{0}^{\eta} h(t) t^{\alpha-\beta-1} d t\right]$, $\forall \mathrm{t}, \mathrm{s} \in[0,1]$.

Proof.

$\left(a_{1}\right)$. For $s \leqslant t$, notice that $\beta \geqslant 1$, we have $(1-s)^{\beta} \leqslant(1-s)$. Therefore,

$$
\begin{aligned}
\mathrm{G}_{1}(\mathrm{t}, \mathrm{s}) & =\frac{1}{\Gamma(\alpha)}\left(\mathrm{t}^{\alpha-1}(1-\mathrm{s})^{\alpha-\beta-1}-(\mathrm{t}-\mathrm{s})^{\alpha-1}\right) \\
& =\frac{1}{\Gamma(\alpha)}\left[\mathrm{t}^{\alpha-1}(1-\mathrm{s})^{\alpha-\beta-1}-\mathrm{t}^{\alpha-1}\left(1-\frac{s}{\mathrm{t}}\right)^{\alpha-1}\right] \\
& \geqslant \frac{1}{\Gamma(\alpha)} \mathrm{t}^{\alpha-1}\left[(1-\mathrm{s})^{\alpha-\beta-1}-(1-\mathrm{s})^{\alpha-\beta-1+\beta}\right] \\
& \geqslant \frac{1}{\Gamma(\alpha)} \mathrm{t}^{\alpha-1}(1-\mathrm{s})^{\alpha-\beta-1}\left[1-(1-\mathrm{s})^{\beta}\right]
\end{aligned}
$$




$$
\geqslant \frac{1}{\Gamma(\alpha)} \mathrm{t}^{\alpha-1}(1-\mathrm{s})^{\alpha-\beta-1}[1-(1-\mathrm{s})]=\frac{1}{\Gamma(\alpha)} \mathrm{t}^{\alpha-1} \mathrm{~s}(1-\mathrm{s})^{\alpha-\beta-1} .
$$

For $s \geqslant t$, we have

$$
\mathrm{G}_{1}(\mathrm{t}, \mathrm{s})=\frac{1}{\Gamma(\alpha)} \mathrm{t}^{\alpha-1}(1-\mathrm{s})^{\alpha-\beta-1} \geqslant \frac{1}{\Gamma(\alpha)} \mathrm{t}^{\alpha-1} \mathrm{~s}(1-\mathrm{s})^{\alpha-\beta-1}
$$

$\left(a_{2}\right)$. For $s \leqslant t$, notice that $\alpha-\beta-1>0$, we have

$$
\begin{aligned}
\mathrm{G}_{1}(\mathrm{t}, \mathrm{s}) & =\frac{1}{\Gamma(\alpha)}\left(\mathrm{t}^{\alpha-1}(1-s)^{\alpha-\beta-1}-(\mathrm{t}-\mathrm{s})^{\alpha-1}\right) \\
& =\frac{1}{\Gamma(\alpha)}(1-s)^{-\beta}\left[(\mathrm{t}(1-\mathrm{s}))^{\alpha-1}-(1-s)^{\beta}(\mathrm{t}-\mathrm{s})^{\alpha-1}\right] \\
& \leqslant \frac{1}{\Gamma(\alpha)}(1-s)^{-\beta}\left[(\mathrm{t}(1-s))^{\alpha-1}-(1-s)^{\alpha-1}(\mathrm{t}-\mathrm{s})^{\alpha-1}\right] \\
& =\frac{1}{\Gamma(\alpha)}(1-s)^{-\beta}(\alpha-1) \int_{(1-s)(t-s)}^{\mathrm{t}(1-s)} x^{\alpha-2} \mathrm{dx} \\
& \leqslant \frac{1}{\Gamma(\alpha)}(1-s)^{-\beta}(\alpha-1) t^{\alpha-2}(1-s)^{\alpha-2}[\mathrm{t}(1-s)-(1-s)(\mathrm{t}-\mathrm{s})] \\
& =\frac{1}{\Gamma(\alpha)}(\alpha-1)(1-s)^{-\beta} \mathrm{t}^{\alpha-2}(1-s)^{\alpha-2} s(1-s) \\
& \leqslant \frac{1}{\Gamma(\alpha)}(\alpha-1) s(1-s)^{\alpha-\beta-1} .
\end{aligned}
$$

For $s \geqslant t$, noticing that $\alpha>2$, we have

$$
\mathrm{G}_{1}(\mathrm{t}, \mathrm{s})=\frac{1}{\Gamma(\alpha)} \mathrm{t}^{\alpha-1}(1-s)^{\alpha-\beta-1} \leqslant \frac{1}{\Gamma(\alpha)} \mathrm{s}^{\alpha-1}(1-\mathrm{s})^{\alpha-\beta-1} \leqslant \frac{1}{\Gamma(\alpha)}(\alpha-1) s(1-s)^{\alpha-\beta-1} .
$$

$\left(a_{3}\right)$. By $\left(a_{2}\right)$ and $\left(a_{1}\right)$, we have

$$
G(t, s)=G_{1}(t, s)+G_{2}(t, s) \leqslant \frac{1}{\Gamma(\alpha)}(\alpha-1) s(1-s)^{\alpha-\beta-1}+\frac{\lambda}{1-\lambda \int_{0}^{\eta} h(t) t^{\alpha-\beta-1} d t} \int_{0}^{\eta} h(t) H(t, s) d t=J(s) .
$$

$\left(a_{4}\right)$. By $\left(a_{1}\right)$ and $\left(a_{3}\right)$, we have

$$
\begin{aligned}
& \mathrm{G}(\mathrm{t}, \mathrm{s})=\mathrm{G}_{1}(\mathrm{t}, \mathrm{s})+\mathrm{G}_{2}(\mathrm{t}, \mathrm{s}) \\
& \geqslant \frac{1}{\Gamma(\alpha)} t^{\alpha-1} s(1-s)^{\alpha-\beta-1}+\frac{\lambda t^{\alpha-1}}{1-\lambda \int_{0}^{\eta} h(t) t^{\alpha-\beta-1} d t} \int_{0}^{\eta} h(t) H(t, s) d t \\
& \geqslant \frac{1}{(\alpha-1)} t^{\alpha-1}\left(\frac{1}{\Gamma(\alpha)}(\alpha-1) s(1-s)^{\alpha-\beta-1}+\frac{\lambda}{1-\lambda \int_{0}^{\eta} h(t) t^{\alpha-\beta-1} d t} \int_{0}^{\eta} h(t) H(t, s) d t\right) \\
& =\frac{1}{(\alpha-1)} \mathrm{t}^{\alpha-1} \mathrm{~J}(\mathrm{~s}) \text {. }
\end{aligned}
$$

On the other hand, by $\left(a_{2}\right)$, for $s \leqslant t$, we have

$$
\begin{aligned}
\mathrm{G}_{1}(\mathrm{t}, \mathrm{s}) & =\frac{1}{\Gamma(\alpha)}\left(\mathrm{t}^{\alpha-1}(1-\mathrm{s})^{\alpha-\beta-1}-(\mathrm{t}-\mathrm{s})^{\alpha-1}\right) \\
& \leqslant \frac{1}{\Gamma(\alpha)}(\alpha-1)(1-s)^{-\beta} \mathrm{t}^{\alpha-2}(1-s)^{\alpha-2} s(1-s) \\
& \leqslant \frac{1}{\Gamma(\alpha)}(\alpha-1)(1-s)^{-\beta} \mathrm{t}^{\alpha-2}(1-s)^{\alpha-2} \mathrm{t}(1-s)
\end{aligned}
$$




$$
=\frac{1}{\Gamma(\alpha)}(\alpha-1) t^{\alpha-1}(1-s)^{\alpha-\beta-1} .
$$

For $\mathrm{t} \leqslant \mathrm{s}$, we have

$$
\mathrm{G}_{1}(\mathrm{t}, \mathrm{s})=\frac{1}{\Gamma(\alpha)} \mathrm{t}^{\alpha-1}(1-\mathrm{s})^{\alpha-\beta-1} \leqslant \frac{1}{\Gamma(\alpha)}(\alpha-1) \mathrm{t}^{\alpha-1}(1-\mathrm{s})^{\alpha-\beta-1}
$$

Therefore,

$$
\begin{aligned}
G(t, s) & =G_{1}(t, s)+G_{2}(t, s) \\
& \leqslant \frac{1}{\Gamma(\alpha)}(\alpha-1) t^{\alpha-1}(1-s)^{\alpha-\beta-1}+\frac{\lambda t^{\alpha-1}}{1-\lambda \int_{0}^{\eta} h(t) t^{\alpha-\beta-1} d t} \int_{0}^{\eta} h(t) H(t, s) d t \\
& \leqslant \frac{1}{\Gamma(\alpha)}(\alpha-1) t^{\alpha-1}(1-s)^{\alpha-\beta-1}+\frac{\lambda t^{\alpha-1}}{1-\lambda \int_{0}^{\eta} h(t) t^{\alpha-\beta-1} d t} \int_{0}^{\eta} h(t) \frac{t^{\alpha-\beta-1}(1-s)^{\alpha-\beta-1}}{\Gamma(\alpha)} d t \\
& =\frac{1}{\Gamma(\alpha)} t^{\alpha-1}(1-s)^{\alpha-\beta-1}\left[(\alpha-1)+\frac{\lambda}{1-\lambda \int_{0}^{\eta} h(t) t^{\alpha-\beta-1} d t} \int_{0}^{\eta} h(t) t^{\alpha-\beta-1} d t\right] .
\end{aligned}
$$

Throughout this article, for notational convenience, denote $e(t)=\frac{1}{(\alpha-1)} t^{\alpha-1}$.

Lemma 2.7. Let $u \in C[0,1]$ satisfy $(2.1)$, where $y \in L^{1}(0,1), y(t) \geqslant 0,0 \leqslant t \leqslant 1$. Then, $u(t) \geqslant e(t)\|u\|, 0 \leqslant$ $t \leqslant 1$.

Proof. By Lemma 2.6, $\frac{1}{(\alpha-1)} t^{\alpha-1} J(s) \leqslant G(t, s) \leqslant J(s)$ for all $t, s \in[0,1]$. Thus, for $0 \leqslant t \leqslant 1$, we get that

$$
u(t)=\int_{0}^{1} G(t, s) y(s) d s \leqslant \int_{0}^{1} J(s) y(s) d s
$$

i.e., $\|u\| \leqslant \int_{0}^{1} J(s) y(s)$ ds. For $0 \leqslant t \leqslant 1$, we have

$$
u(t)=\int_{0}^{1} G(t, s) y(s) d s \geqslant \frac{1}{(\alpha-1)} t^{\alpha-1} \int_{0}^{1} J(s) y(s) d s \geqslant \frac{1}{(\alpha-1)} t^{\alpha-1}\|u\| .
$$

Lemma 2.8. Let $w(t) \in C[0,1]$ satisfy

$$
\left\{\begin{array}{l}
D_{0+}^{\alpha} w(t)+L(t)=0,0<t<1 \\
w(0)=w^{\prime}(0)=\cdots=w^{(n-2)}(0)=0, \quad D_{0+}^{\beta} w(1)=\lambda \int_{0}^{\eta} h(t) D_{0+}^{\beta} w(t) d t
\end{array}\right.
$$

where $\mathrm{L} \in \mathrm{L}^{1}(0,1), \mathrm{L}(\mathrm{t})>0$. Then, $w(\mathrm{t}) \leqslant \mathrm{C}\|\mathrm{L}\|_{1} \mathrm{e}(\mathrm{t}), 0 \leqslant \mathrm{t} \leqslant 1$, here

$$
C=\frac{1}{\Gamma(\alpha)}\left[(\alpha-1)^{2}+\frac{\lambda(\alpha-1)}{1-\lambda \int_{0}^{\eta} h(t) t^{\alpha-\beta-1} d t} \cdot \int_{0}^{\eta} h(t) t^{\alpha-\beta-1} d t\right] .
$$

Proof. For any $0 \leqslant \mathrm{t} \leqslant 1$, noticing that $\beta>1, \alpha-\beta-1>0, n-1<\alpha \leqslant n, n \geqslant 3$, by Lemma 2.6, one has

$$
\begin{aligned}
w(t) & =\int_{0}^{1} G(t, s) L(s) d s \\
& \leqslant \frac{1}{\Gamma(\alpha)}\left[(\alpha-1)+\frac{\lambda}{1-\lambda \int_{0}^{\eta} h(t) t^{\alpha-\beta-1} d t} \int_{0}^{\eta} h(t) t^{\alpha-\beta-1} d t\right] t^{\alpha-1} \int_{0}^{1}(1-s)^{\alpha-\beta-1} L(s) d s
\end{aligned}
$$




$$
\begin{aligned}
& \leqslant \frac{1}{\Gamma(\alpha)}\left[(\alpha-1)+\frac{\lambda}{1-\lambda \int_{0}^{\eta} h(t) t^{\alpha-\beta-1} d t} \int_{0}^{\eta} h(t) t^{\alpha-\beta-1} d t\right] t^{\alpha-1} \int_{0}^{1} L(s) d s \\
& =\frac{1}{\Gamma(\alpha)}\left[(\alpha-1)^{2}+\frac{\lambda(\alpha-1)}{1-\lambda \int_{0}^{\eta} h(t) t^{\alpha-\beta-1} d t} \int_{0}^{\eta} h(t) t^{\alpha-\beta-1} d t\right]\|L\|_{1} \frac{1}{\alpha-1} t^{\alpha-1} \\
& =C\|L\|_{1} e(t) .
\end{aligned}
$$

Hence, we have proved that $w(t) \leqslant C\|L\|_{1} e(t), 0 \leqslant t \leqslant 1$.

Lemma 2.9 ([16]). Let $\Omega_{1}$ and $\Omega_{2}$ be two bounded open sets in Banach space $E$ such that $\theta \in \Omega_{1}$ and $\bar{\Omega}_{1} \subset$ $\Omega_{2}, \mathrm{~A}: \mathrm{P} \cap\left(\bar{\Omega}_{2} \backslash \Omega_{1}\right) \rightarrow \mathrm{P}$ a completely continuous operator, where $\theta$ denotes the zero element of $\mathrm{E}$ and $\mathrm{P}$ a cone of

E. Suppose that one of the following conditions holds

(i) $\|A u\| \leqslant\|u\|, \forall u \in P \cap \partial \Omega_{1} ; \quad\|A u\| \geqslant\|u\|, \forall u \in P \cap \partial \Omega_{2} ;$

(ii) $\|A u\| \geqslant\|u\|, \forall u \in P \cap \partial \Omega_{1} ; \quad\|A u\| \leqslant\|u\|, \forall u \in P \cap \partial \Omega_{2}$.

Then $\mathrm{A}$ has a fixed point in $\mathrm{P} \cap\left(\bar{\Omega}_{2} \backslash \Omega_{1}\right)$.

\section{Main results}

Let

$$
\begin{aligned}
& P=\{u \in E: u(t) \geqslant 0, t \in[0,1]\}, \\
& K=\{u: u \in P, u(t) \geqslant e(t)\|u\|, t \in[0,1]\} .
\end{aligned}
$$

Obviously, $\mathrm{K}$ is a subcone of $\mathrm{P}$ in Banach space $\mathrm{E}$ and $(\mathrm{E}, \mathrm{K})$ is an ordering Banach space.

3.1. Case one: $\mathrm{f}$ is singular with respect to the time variable

$\left(\mathrm{H}_{0}\right) \mathrm{f} \in \mathrm{C}((0,1) \times[0,+\infty),(-\infty,+\infty))$, there exists a function $\mathrm{L} \in \mathrm{L}^{1}(0,1), \mathrm{L}(\mathrm{t})>0$, such that $f(t, u) \geqslant$ $-\mathrm{L}(\mathrm{t})$ for all $\mathrm{t} \in(0,1), \mathrm{u} \geqslant 0$;

$\left(\mathrm{A}_{1}\right)$ for any $r>0$, there exists a nonnegative function $\gamma_{r} \in \mathrm{C}(0,1) \cap \mathrm{L}^{1}(0,1)$ such that $|\mathrm{f}(\mathrm{t}, \mathrm{u})| \leqslant$ $\gamma_{\mathrm{r}}(\mathrm{t}),(\mathrm{t}, \mathrm{u}) \in(0,1) \times[0, \mathrm{r}]$;

$\left(A_{2}\right)$ there exists an $r_{1}>C\|L\|_{1}$ such that

$$
\int_{0}^{1} J(s) \varphi\left(s, r_{1}\right) d s<r_{1}
$$

where $\varphi\left(t, r_{1}\right)=\max \left\{f(t, u): 0 \leqslant u \leqslant r_{1}\right\}+L(t), 0<t<1 ;$

$\left(A_{3}\right)$ there exist two positive numbers $0<a<b \leqslant 1$ and $r_{2}>r_{1}$ such that

$$
\frac{1}{\alpha-1} \int_{a}^{b} J(s) \psi\left(s, r_{2}\right) d s>r_{2}
$$

where $\psi\left(t, r_{2}\right)=\min \left\{f(t, u):\left(r_{2}-C\|L\|_{1}\right) \sigma \leqslant u \leqslant r_{2}, \sigma=\frac{1}{\alpha-1} a^{\alpha-1}\right\}+L(t), a \leqslant t \leqslant b$.

Theorem 3.1. Suppose that conditions $\left(\mathrm{H}_{0}\right),\left(\mathrm{A}_{1}\right),\left(\mathrm{A}_{2}\right)$, and $\left(\mathrm{A}_{3}\right)$ hold. Then BVP (1.1)-(1.2) has at least one positive solution.

Proof. We first consider the following modified boundary value problems (MBVP for short):

$$
\left\{\begin{array}{l}
D_{0+}^{\alpha} u(t)+F^{*}(t, u(t)-w(t))=0, \quad 0<t<1, \\
u(0)=u^{\prime}(0)=\cdots=u^{(n-2)}(0)=0, \quad D_{0+}^{\beta}(1)=\lambda \int_{0}^{\eta} h(t) D_{0+}^{\beta} u(t) d t,
\end{array}\right.
$$


here,

$$
F^{*}(t, u)=H^{*}(t, u)+L(t), \quad H^{*}(t, u)=\left\{\begin{array}{ll}
f(t, u), & u \geqslant 0, \\
f(t, 0), & u<0,
\end{array} \quad t \in(0,1) .\right.
$$

Define another operator $\mathrm{T}^{*}$ as follows

$$
\left(T^{*} u\right)(t)=\int_{0}^{1} G(t, s) F^{*}(s, u(s)-w(s)) d s, 0 \leqslant t \leqslant 1 .
$$

Then, $u \in C[0,1]$ is a solution of (3.1) if and only if $u \in C[0,1]$ is a fixed point of the operator $T^{*}$.

In the following, we divide the proof into three parts.

(I) We shall show that $\mathrm{T}^{*}: \mathrm{K} \rightarrow \mathrm{K}$ is completely continuous.

For any $u \in K$, by $\left(H_{0}\right)$ and $\left(A_{1}\right)$, we can see that $T^{*} u \in C[0,1]$. If $u_{n} \in K$ and $\left\|u_{n}-u\right\| \rightarrow 0$ as $n \rightarrow \infty(n=1,2, \cdots)$, then for any $t \in(0,1)$, we have

$$
\lim _{n \rightarrow \infty} F^{*}\left(t, u_{n}(t)-w(t)\right)=F^{*}(t, u(t)-w(t))
$$

Let $\bar{r}_{1}=\sup _{n}\left\|u_{n}\right\|+\|w\|+1$. By $\left(A_{1}\right)$, there exists a nonnegative function $\gamma_{\bar{r}_{1}} \in L^{1}(0,1)$ such that

$$
\left|F^{*}\left(t, u_{n}(t)-w(t)\right)-F^{*}(t, u(t)-w(t))\right| \leqslant 2 \gamma_{\bar{r}_{1}}(t), 0<t<1 .
$$

It follows from the Lebesgue's dominated convergence theorem that

$$
\begin{aligned}
\lim _{n \rightarrow \infty}\left\|T^{*} u_{n}-T^{*} u\right\| & \leqslant \lim _{n \rightarrow \infty} \max _{0 \leqslant t \leqslant 1} \int_{0}^{1} G(t, s)\left|F^{*}\left(s, u_{n}(s)-w(s)\right)-F^{*}(s, u(s)-w(s))\right| d s \\
& \leqslant \max _{0 \leqslant t \leqslant 1} G(t, s) \lim _{n \rightarrow \infty} \int_{0}^{1}\left|F^{*}\left(s, u_{n}(s)-w(s)\right)-F^{*}(s, u(s)-w(s))\right| d s \\
& \leqslant \max _{0 \leqslant t \leqslant 1} G(t, s) \int_{0}^{1} \lim _{n \rightarrow \infty}\left|F^{*}\left(s, u_{n}(s)-w(s)\right)-F^{*}(s, u(s)-w(s))\right| d s=0 .
\end{aligned}
$$

Hence, $T^{*}: K \rightarrow C[0,1]$ is continuous.

Let $D \subset K$ be any bounded set and $\bar{r}_{2}=\sup \{\|u\|: u \in D\}+\|w\|+1$. For $u \in D$, by $\left(A_{1}\right)$, there exists a nonnegative function $\gamma_{\overline{\mathrm{r}}_{2}} \in \mathrm{L}^{1}(0,1)$ such that

$$
\left|H^{*}(t, u(t)-w(t))\right| \leqslant \gamma_{\bar{r}_{2}}(t), 0<t<1
$$

As a consequence,

$$
\begin{aligned}
\left\|T^{*} u\right\| & =\max _{0 \leqslant t \leqslant 1} \int_{0}^{1} G(t, s) F^{*}(s, u(s)-w(s)) d s \\
& \leqslant \max _{0 \leqslant t, s \leqslant 1} G(t, s) \int_{0}^{1}\left[H^{*}(s, u(s)-w(s))+L(s)\right] d s \\
& \leqslant \max _{0 \leqslant t, s \leqslant 1} G(t, s) \int_{0}^{1}\left[\gamma_{\bar{r}_{2}}(s)+L(s)\right] d s<+\infty,
\end{aligned}
$$

which means that $T^{*}(D) \subset C[0,1]$ is uniformly bounded.

Since $G(t, s)$ is continuous on $[0,1] \times[0,1]$, it is also uniformly continuous on $[0,1] \times[0,1]$ as well. For any fixed $s \in[0,1]$ and any $\varepsilon>0$, there exists a constant $\delta>0$ such that for $t_{1}, t_{2} \in[0,1]$ with $\left|t_{1}-t_{2}\right|<\delta$

$$
\left|G\left(t_{1}, s\right)-G\left(t_{2}, s\right)\right|<\frac{\varepsilon}{\int_{0}^{1}\left[\gamma_{\bar{r}_{2}}(s)+L(s)\right] d s} .
$$


Thus, for any $u \in D$,

$$
\begin{aligned}
\left|T^{*} u\left(t_{1}\right)-T^{*} u\left(t_{2}\right)\right| & =\int_{0}^{1}\left|G\left(t_{1}, s\right)-G\left(t_{2}, s\right)\right| F^{*}(s, u(s)-w(s)) d s \\
& \leqslant \frac{\varepsilon}{\int_{0}^{1}\left[\gamma_{\bar{r}_{2}}(s)+L(s)\right] d s} \cdot \int_{0}^{1}\left[\gamma_{\bar{r}_{2}}(s)+L(s)\right] d s=\varepsilon,
\end{aligned}
$$

which shows that $T^{*}(D) \subset C[0,1]$ is equicontinuous. Thus, Arzelà-Ascoli theorem guarantees that $T^{*}$ : $\mathrm{K} \rightarrow \mathrm{C}[0,1]$ is completely continuous.

On the other hand, for any $u \in K$ with $\|u\|=r, t \in[0,1]$, by Lemma 2.6 and $\left(A_{1}\right)$, we have

$$
\begin{aligned}
\left(T^{*} \mathrm{u}\right)(\mathrm{t}) & =\int_{0}^{1} \mathrm{G}(\mathrm{t}, \mathrm{s}) \mathrm{F}^{*}(\mathrm{~s}, \mathrm{u}(\mathrm{s})-w(\mathrm{~s})) \mathrm{d} s \leqslant \int_{0}^{1} \mathrm{~J}(\mathrm{~s}) \mathrm{F}^{*}(\mathrm{~s}, \mathrm{u}(\mathrm{s})-w(\mathrm{~s})) \mathrm{d} s \\
& =\int_{0}^{1} \mathrm{~J}(\mathrm{~s})\left[\mathrm{H}^{*}(\mathrm{~s}, \mathrm{u}(\mathrm{s})-w(\mathrm{~s}))+\mathrm{L}(\mathrm{s})\right] \mathrm{d} s<\int_{0}^{1} \mathrm{~J}(\mathrm{~s})\left[\gamma_{\mathrm{r}}(\mathrm{s})+\mathrm{L}(\mathrm{s})\right] \mathrm{d} \mathrm{s}<+\infty .
\end{aligned}
$$

Hence,

$$
\left\|T^{*} u\right\| \leqslant \int_{0}^{1} J(s) F(s, u(s)-w(s)) d s .
$$

For any $u \in K$, it follows from Lemma 2.6 that

$$
\begin{aligned}
\left(T^{*} u\right)(t) & =\int_{0}^{1} G(t, s) F(s, u(s)-w(s)) d s \\
& \geqslant \frac{1}{(\alpha-1)} t^{\alpha-1} \int_{0}^{1} J(s) F(s, u(s)-w(s)) d s \geqslant \frac{1}{(\alpha-1)} t^{\alpha-1}\left\|T^{*} u\right\|=e(t)\left\|T^{*} u\right\|,
\end{aligned}
$$

which means that $\mathrm{T}^{*}: \mathrm{K} \rightarrow \mathrm{K}$.

(II) We shall show the existence of positive solutions for MBVP (3.1).

If $u \in \Omega\left(r_{1}\right)$, then we have

$$
F^{*}(t, u(t)-w(t)) \leqslant \varphi\left(t, r_{1}\right), 0<t<1
$$

Following Lemma $2.6\left(a_{3}\right)$, and $\left(A_{2}\right)$, we get that

$$
\left\|T^{*} u\right\|=\max _{0 \leqslant t \leqslant 1} \int_{0}^{1} G(t, s) F^{*}(s, u(s)-w(s)) d s \leqslant \int_{0}^{1} J(s) \varphi\left(s, r_{1}\right) d s<r_{1} .
$$

If $u \in \partial \Omega\left(r_{2}\right)$, then $\|u\|=r_{2}$ and $u(t) \geqslant r_{2} e(t), 0 \leqslant t \leqslant 1$. Thus, we get

$$
r_{2} \geqslant u(t)-w(t) \geqslant\left(r_{2}-C\|L\|_{1}\right) e(t) \geqslant 0,0 \leqslant t \leqslant 1,
$$

and

$$
r_{2} \geqslant u(t)-w(t) \geqslant\left(r_{2}-C\|L\|_{1}\right) e(t) \geqslant\left(r_{2}-C\|L\|_{1}\right) \sigma, a \leqslant t \leqslant b .
$$

By the definition of $\psi\left(t, r_{2}\right)$, one has

$$
f(t, u(t)-w(t))+L(t) \geqslant \psi\left(t, r_{2}\right), a \leqslant t \leqslant b .
$$

By Lemma $2.6\left(a_{4}\right),\left(A_{3}\right)$, and (3.3), we have

$$
\left\|T^{*} u\right\|=\max _{0 \leqslant t \leqslant 1} \int_{0}^{1} G(t, s) F(s, u(s)-w(s)) d s
$$




$$
\geqslant \frac{1}{\alpha-1} \max _{0 \leqslant t \leqslant 1} \int_{0}^{1} t^{\alpha-1} J(s)[f(s, u(s)-w(s))+L(s)] d s \geqslant \frac{1}{\alpha-1} \int_{a}^{b} J(s) \psi\left(s, r_{2}\right) \geqslant r_{2},
$$

i.e.,

$$
\left\|T^{*} u\right\| \geqslant\|u\|, \forall u \in \partial \Omega\left(r_{2}\right) .
$$

It follows from (3.2), (3.4), and Lemma 2.9 that $\mathrm{T}^{*}$ has at least one fixed point $\widehat{u} \in \bar{\Omega}_{\mathrm{r}_{2}} \backslash \Omega_{\mathrm{r}_{1}}$.

(III) We shall show that $\widehat{u}(t)>w(t), t \in[0,1]$. For any $t \in[0,1], \widehat{u}(t) \geqslant\|\widehat{u}\| e(t) \geqslant r_{1} e(t)>C\|L\|_{1} e(t) \geqslant$ $w(t)$. Let $u(t)=\widehat{u}(t)-w(t)$, then $u(t)$ is a positive solution for BVP (1.1)-(1.2).

3.2. Case two: $f$ is singular with respect to both the time variable and the space variable

$\left(H_{1}\right) f \in C((0,1) \times(0,+\infty),(-\infty,+\infty))$, there exists a function $L \in L^{1}(0,1), L(t)>0$, such that $f(t, u) \geqslant$ $-\mathrm{L}(\mathrm{t})$ for all $\mathrm{t} \in(0,1), \mathrm{u}>0$;

$\left(B_{1}\right)$ for any positive numbers $r_{1}<r_{2}$, there exists a nonnegative continuous function $\gamma_{r_{1}, r_{2}} \in L^{1}(0,1)$ such that

$$
|f(t, u)| \leqslant \gamma_{r_{1}, r_{2}}(t), 0<t<1, r_{1} e(t) \leqslant u \leqslant r_{2}
$$

with

$$
\int_{0}^{1} s(1-s)^{\alpha-\beta-1} \gamma_{\mathrm{r}_{1}, \mathrm{r}_{2}}(\mathrm{~s}) \mathrm{d} \mathrm{s}<+\infty ;
$$

$\left(B_{2}\right)$ there exists an $\widehat{r}_{1}>C\|L\|_{1}$ such that

$$
\int_{0}^{1} \mathrm{~J}(\mathrm{~s}) \varphi\left(\mathrm{s}, \widehat{\mathrm{r}}_{1}\right) \mathrm{d} s<\widehat{\mathrm{r}}_{1},
$$

where $\varphi\left(t, \widehat{r}_{1}\right)=\max \left\{f(t, u):\left(\widehat{r}_{1}-C\|L\|_{1}\right) e(t) \leqslant u \leqslant \widehat{r}_{1}\right\}+L(t) ;$

$\left(B_{3}\right)$ there exists $\widehat{r}_{2}>\widehat{r}_{1}$ such that

$$
\int_{0}^{1} \frac{1}{(\alpha-1)} J(s) \psi\left(s, \widehat{r}_{2}\right) d s>\widehat{r}_{2},
$$

where $\psi\left(t, \widehat{r}_{2}\right)=\min \left\{f(t, u):\left(\widehat{r}_{2}-C\|L\|_{1}\right) e(t) \leqslant u \leqslant \widehat{r}_{2}\right\}+L(t)$.

Theorem 3.2. Suppose that conditions $\left(\mathrm{H}_{1}\right),\left(\mathrm{B}_{1}\right),\left(\mathrm{B}_{2}\right)$, and $\left(\mathrm{B}_{3}\right)$ hold. Then BVP (1.1)-(1.2) has at least one positive solution.

Proof. We first consider the following modified approximating BVP (MABVP for short)

$$
\left\{\begin{array}{l}
D_{0+}^{\alpha} u(t)+F_{n}^{*}(t, u(t)-w(t))=0,0<t<1, \\
u(0)=u^{\prime}(0)=\cdots=u^{(n-2)}(0)=0, \quad D_{0+}^{\beta}(1)=\lambda \int_{0}^{\eta} h(t) D_{0+}^{\beta} u(t) d t
\end{array}\right.
$$

here,

$$
F_{n}^{*}(t, u)=H_{n}^{*}(t, u)+L(t), \quad H_{n}^{*}(t, u)=\left\{\begin{array}{ll}
f(t, u), & u \geqslant \frac{1}{n}, \\
f\left(t, \frac{1}{n}\right), & u<\frac{1}{n},
\end{array} \quad t \in(0,1)\right.
$$

Define an operator $T_{n}^{*}$ as follows

$$
\left(T_{n}^{*} u\right)(t)=\int_{0}^{1} G(t, s) F_{n}^{*}(s, u(s)-w(s)) d s, 0 \leqslant t \leqslant 1
$$

In the following, we divide the proof into three parts.

(I) For any $\mathrm{C}\|\mathrm{L}\|_{1}<\mathrm{r}_{1}<\mathrm{r}_{2}$, we prove that $\mathrm{T}_{\mathrm{n}}^{*}: \mathrm{K} \cap\left(\Omega_{\mathrm{r}_{2}} \backslash \Omega_{\mathrm{r}_{1}}\right) \rightarrow \mathrm{K}, \mathrm{n}>\frac{1}{\mathrm{r}_{1}}$ is completely continuous.

Notice that $u(t)-w(t) \geqslant\left(r_{1}-C\|L\|_{1}\right) e(t)>0,0<t<1 ; n>\frac{1}{r_{1}}$, we have

$$
\left(r_{1}-C\|L\|_{1}\right) e(t) \leqslant \max \left\{u(t)-w(t), \frac{1}{n}\right\} \leqslant r_{2} .
$$


Thus, it follows from $\left(\mathrm{H}_{1}\right),\left(\mathrm{B}_{1}\right)$, and Lemma $2.4\left(\mathrm{a}_{3}\right)$ and $\left(\mathrm{a}_{4}\right)$ that

$$
\begin{aligned}
& \left(T_{n}^{*} u\right)(t)=\int_{0}^{1} G(t, s) F_{n}^{*}(s, u(s)-w(s)) d s \\
& \leqslant \int_{0}^{1} \mathrm{~J}(\mathrm{~s})\left[\mathrm{H}_{\mathrm{n}}^{*}(\mathrm{~s}, \mathrm{u}(\mathrm{s})-w(\mathrm{~s}))+\mathrm{L}(\mathrm{s})\right] \mathrm{d} s \\
& =\int_{0}^{1}\left[\frac{1}{\Gamma(\alpha)}(\alpha-1) s(1-s)^{\alpha-\beta-1}\right. \\
& \left.+\frac{\lambda}{1-\lambda \int_{0}^{\eta} h(t) t^{\alpha-\beta-1} d t} \int_{0}^{\eta} h(t) H(t, s) d t\right]\left[H_{n}^{*}(s, u(s)-w(s))+L(s)\right] d s \\
& \leqslant \int_{0}^{1}\left[\frac{1}{\Gamma(\alpha)}(\alpha-1) s(1-s)^{\alpha-\beta-1}\right. \\
& \left.+\frac{\lambda}{\Gamma(\alpha)\left(1-\lambda \int_{0}^{\eta} h(t) t^{\alpha-\beta-1} d t\right)} \int_{0}^{\eta} h(t) t^{\alpha-\beta-1} d t\right]\left[H_{n}^{*}(s, u(s)-w(s))+L(s)\right] d s \\
& \leqslant \int_{0}^{1}\left[\frac{1}{\Gamma(\alpha)}(\alpha-1) s(1-s)^{\alpha-\beta-1}\right. \\
& \left.+\frac{\lambda}{\Gamma(\alpha)\left(1-\lambda \int_{0}^{\eta} h(t) t^{\alpha-\beta-1} d t\right)} \int_{0}^{\eta} h(t) t^{\alpha-\beta-1} d t\right]\left[\gamma_{r_{1}-C\|L\|_{1}, r_{2}}(s)+L(s)\right] d s<+\infty .
\end{aligned}
$$

So, $\mathrm{T}_{\mathrm{n}}^{*}: \mathrm{K} \cap\left(\Omega_{\mathrm{r}_{2}} \backslash \Omega_{\mathrm{r}_{1}}\right) \rightarrow \mathrm{E}$ is well-defined.

On the other hand, for any $u \in K \cap\left(\Omega_{r_{2}} \backslash \Omega_{r_{1}}\right), t \in[0,1]$, one gets from (3.6) and Lemma 2.6

$$
\left(\mathrm{T}_{\mathrm{n}}^{*} \mathrm{u}\right)(\mathrm{t})=\int_{0}^{1} \mathrm{G}(\mathrm{t}, \mathrm{s}) \mathrm{F}_{\mathrm{n}}^{*}(\mathrm{~s}, \mathrm{u}(\mathrm{s})-w(\mathrm{~s})) \mathrm{d} s \leqslant \int_{0}^{1} \mathrm{~J}(\mathrm{~s}) \mathrm{F}_{\mathrm{n}}^{*}(\mathrm{~s}, \mathrm{u}(\mathrm{s})-w(\mathrm{~s})) \mathrm{d} s<+\infty .
$$

Hence,

$$
\left\|\mathrm{T}_{\mathrm{n}}^{*} \mathrm{u}\right\| \leqslant \int_{0}^{1} \mathrm{G}(\mathrm{t}, \mathrm{s}) \mathrm{F}_{\mathrm{n}}^{*}(\mathrm{~s}, \mathrm{u}(\mathrm{s})-w(\mathrm{~s})) \mathrm{d} \mathrm{s} .
$$

For any $u \in K$, it follows from Lemma 2.6 that

$$
\begin{aligned}
\left(T_{n}^{*} u\right)(t) & =\int_{0}^{1} G(t, s) F_{n}^{*}(s, u(s)-w(s)) d s \\
& \geqslant \frac{1}{(\alpha-1)} t^{\alpha-1} \int_{0}^{1} J(s) F_{n}^{*}(s, u(s)-w(s)) d s \geqslant \frac{1}{(\alpha-1)} t^{\alpha-1}\left\|T_{n}^{*} u\right\|=e(t)\left\|T_{n}^{*} u\right\|,
\end{aligned}
$$

which means that $\mathrm{T}_{\mathrm{n}}^{*}: \mathrm{K} \cap\left(\Omega_{\mathrm{r}_{2}} \backslash \Omega_{\mathrm{r}_{1}}\right) \rightarrow \mathrm{K}$.

Let $u_{n}, \bar{u} \in K \cap\left(\Omega_{r_{2}} \backslash \Omega_{r_{1}}\right),\left\|u_{n}-\bar{u}\right\| \rightarrow 0(n \rightarrow \infty)$, then $\lim _{n \rightarrow \infty} u_{n}(t)=\bar{u}(t), t \in(0,1)$. Let

$$
\begin{aligned}
& \left(T_{n}^{1} u\right)(t)=F_{n}^{*}(t, u(t)-w(t)), 0<t<1, u \in K \cap\left(\Omega_{\left.r_{2} \backslash \Omega_{r_{1}}\right),}\right. \\
& \left(T_{n}^{2} u\right)(t)=\int_{0}^{1} G(t, s) u(s) d s, 0<t<1, u \in L^{1}(0,1) .
\end{aligned}
$$

By $\left(\mathrm{H}_{1}\right)$, we have

$$
\lim _{n \rightarrow \infty} F_{n}^{*}\left(t, u_{n}(t)-w(t)\right)=F_{n}^{*}(t, \bar{u}(t)-w(t)), 0<t<1 .
$$

For $u_{n}, \bar{u} \in K \cap\left(\Omega_{r_{2}} \backslash \Omega_{r_{1}}\right)$, by $\left(H_{1}\right)$ and $\left(B_{1}\right)$, we know that

$$
\begin{gathered}
\mathrm{F}_{\mathrm{n}}^{*}\left(\mathrm{t}, \mathrm{u}_{\mathrm{n}}(\mathrm{t})-w(\mathrm{t})\right) \leqslant \gamma_{\mathrm{r}_{1}-\mathrm{C}\|\mathrm{L}\|_{1}, \mathrm{r}_{2}}(\mathrm{t})+\mathrm{L}(\mathrm{t}), 0<\mathrm{t}<1, \\
\mathrm{~F}_{\mathrm{n}}^{*}(\mathrm{t}, \overline{\mathrm{u}}(\mathrm{t})-w(\mathrm{t})) \leqslant \gamma_{\mathrm{r}_{1}-\mathrm{C}\|\mathrm{L}\|_{1}, \mathrm{r}_{2}}(\mathrm{t})+\mathrm{L}(\mathrm{t}), 0<\mathrm{t}<1 .
\end{gathered}
$$


Thus, we have

$$
\left|F_{n}^{*}\left(t, u_{n}(t)-w(t)\right)-F_{n}^{*}(t, \bar{u}(t)-w(t))\right| \leqslant 2 \gamma_{r_{1}-C\|L\|_{1}, r_{2}}(t) .
$$

It is easy to see from (3.7), (3.8), and Lebesgue dominated convergence theorem that

$$
\lim _{n \rightarrow \infty} \int_{0}^{1}\left|\left(T_{n}^{1}\right) u_{n}(t)-\left(T_{n}^{1}\right) \bar{u}(t)\right| d t=0,
$$

which means that $\mathrm{T}_{\mathfrak{n}}^{1}: \mathrm{K} \cap\left(\Omega_{\mathrm{r}_{2}} \backslash \Omega_{\mathrm{r}_{1}}\right) \rightarrow \mathrm{L}^{1}[0,1]$ is continuous. We have from Arzelà-Ascoli theorem that $\mathrm{T}_{\mathrm{n}}^{2}: \mathrm{L}^{1}[0,1] \rightarrow \mathrm{C}[0,1]$ is completely continuous. As a result, $\mathrm{T}_{\mathrm{n}}^{*}=\mathrm{T}_{\mathrm{n}}^{2} \circ \mathrm{T}_{\mathrm{n}}^{1}: \mathrm{K} \cap\left(\Omega_{\mathrm{r}_{2}} \backslash \Omega_{\mathrm{r}_{1}}\right) \rightarrow \mathrm{K}$ is completely continuous.

(II) We show the existence of positive solution of MABVP (3.5) for sufficiently large $n$.

If $u \in K \cap \partial \Omega\left(\widehat{r}_{1}\right)$, then $\|u\|=\widehat{r}_{1}$ and $u(t) \geqslant \widehat{r}_{1} e(t), 0 \leqslant t \leqslant 1$. Thus, for $n>\frac{1}{\widehat{r}_{1}}$, we get

$$
\left(\widehat{r}_{1}-\mathrm{C}\|\mathrm{L}\|_{1}\right) e(\mathrm{t}) \leqslant \max \left\{\mathrm{u}(\mathrm{t})-w(\mathrm{t}), \frac{1}{\mathrm{n}}\right\} \leqslant \widehat{r}_{1} .
$$

By the definition of $\varphi\left(t, \widehat{r}_{1}\right)$, one has

$$
\mathrm{H}_{\mathrm{n}}^{*}(\mathrm{t}, \mathrm{u}(\mathrm{t})-w(\mathrm{t}))+\mathrm{L}(\mathrm{t}) \leqslant \varphi\left(\mathrm{t}, \widehat{r}_{1}\right) .
$$

By $\left(B_{2}\right)$ and Lemma 2.6, we have

$$
\begin{aligned}
\left\|\mathrm{T}_{n}^{*} \mathrm{u}\right\| & =\max _{0 \leqslant \mathrm{t} \leqslant 1} \int_{0}^{1} \mathrm{G}(\mathrm{t}, \mathrm{s}) \mathrm{F}_{\mathrm{n}}^{*}(\mathrm{~s}, \mathrm{u}(\mathrm{s})-w(\mathrm{~s})) \mathrm{d} s \\
& =\max _{0 \leqslant \mathrm{t} \leqslant 1} \int_{0}^{1} \mathrm{G}(\mathrm{t}, \mathrm{s})\left[\mathrm{H}_{\mathrm{n}}^{*}(\mathrm{~s}, \mathrm{u}(\mathrm{s})-w(\mathrm{~s}))+\mathrm{L}(\mathrm{s})\right] \mathrm{d} s \\
& \leqslant \int_{0}^{1} \mathrm{~J}(\mathrm{~s}) \varphi\left(\mathrm{s}, \widehat{\mathrm{r}}_{1}\right) \mathrm{d} s<\widehat{r}_{1},
\end{aligned}
$$

i.e.,

$$
\left\|\mathrm{T}_{\mathrm{n}}^{*} \mathrm{u}\right\| \leqslant\|u\|, \forall u \in \mathrm{K} \cap \partial \Omega\left(\widehat{r}_{1}\right) .
$$

If $u \in K \cap \partial \Omega\left(\widehat{r}_{2}\right)$, then $\|u\|=\widehat{r}_{2}$ and $u(t) \geqslant \widehat{r}_{2} e(t), 0 \leqslant t \leqslant 1$. Thus, for $n>\frac{1}{\widehat{r}_{2}}$, we get

$$
\widehat{r}_{2} \geqslant \max \left\{u(t)-w(t), \frac{1}{n}\right\} \geqslant\left(\widehat{r}_{2}-C\|L\|_{1}\right) e(t) .
$$

By the definition of $\psi\left(t, \widehat{r}_{2}\right)$, one has

$$
H_{n}^{*}(t, u(t)-w(t))+L(t) \geqslant \psi\left(t, \widehat{r}_{2}\right) .
$$

By Lemma 2.6, $\left(\mathrm{B}_{3}\right)$, and (3.10), we have

$$
\begin{aligned}
\left\|\mathrm{T}_{\mathrm{n}}^{*} \mathrm{u}\right\| & =\max _{0 \leqslant \mathrm{t} \leqslant 1} \int_{0}^{1} \mathrm{G}(\mathrm{t}, \mathrm{s}) \mathrm{F}_{\mathrm{n}}^{*}(\mathrm{~s}, \mathrm{u}(\mathrm{s})-w(\mathrm{~s})) \mathrm{d} s \\
& \geqslant \max _{0 \leqslant \mathrm{t} \leqslant 1} \int_{0}^{1} \frac{1}{(\alpha-1)} \mathrm{t}^{\alpha-1} \mathrm{~J}(\mathrm{~s})\left[\mathrm{H}_{\mathrm{n}}^{*}(\mathrm{~s}, \mathrm{u}(\mathrm{s})-w(\mathrm{~s}))+\mathrm{L}(\mathrm{s})\right] \mathrm{d} s \\
& \geqslant \int_{0}^{1} \frac{1}{(\alpha-1)} \mathrm{J}(\mathrm{s}) \psi\left(\mathrm{s}, \widehat{\mathrm{r}}_{2}\right) \mathrm{d} \mathrm{s} \geqslant \widehat{\mathrm{r}}_{2},
\end{aligned}
$$

i.e.,

$$
\left\|\mathrm{T}_{\mathrm{n}}^{*} \mathrm{u}\right\| \geqslant\|u\|, \forall u \in K \cap \partial \Omega\left(\widehat{r}_{2}\right) .
$$

Take $m_{0}=\max \left\{\frac{1}{\widehat{r}_{1}}, \frac{1}{\widehat{r}_{2}}\right\}$. Let $N=\left\{m_{0}, m_{0}+1, \cdots\right\}$. Then, for $n \in N$, both (3.9) and (3.11) hold. This together with Lemma 2.9 shows that $T_{n}^{*}(n \in N)$ has at least one fixed point $\widehat{u_{n}} \in K \cap \bar{\Omega}_{\widehat{r}_{2}} \backslash \Omega_{\widehat{r}_{1}}$. 
(III) In a similar manner, we can show that $\widehat{u_{n}}(t)>w(t), n>N, t \in[0,1]$ and satisfies

$$
\widehat{u_{n}}(t)=\int_{0}^{1} G(t, s)\left[H_{n}^{*}\left(s, \widehat{u_{n}}(s)-w(s)\right)+L(s)\right] d s, 0<t<1 .
$$

By $\left(B_{1}\right)$, we know $\left\{\widehat{u_{n}}: n>N\right\}$ is a bounded, equicontinuous family on $[0,1]$. Arzelà-Ascoli theorem guarantees the existence of a subsequence of $N_{0}$ of $N$ and a function $\widehat{u} \in C[0,1]$ with $\widehat{u_{n}}$ converging uniformly on $[0,1]$ to $\widehat{u}$ as $n \rightarrow \infty$ through $N_{0}$. Let $n \rightarrow \infty$ on both sides of (3.12), considering the fact $\widehat{u_{n}}(t) \geqslant e(t)\left\|\widehat{u_{n}}\right\| \geqslant \widehat{r}_{1} e(t)$, we get

$$
\widehat{u}(t)=\int_{0}^{1} G(t, s)[f(s, \widehat{u}(s)-w(s))+L(s)] d s, 0<t<1 .
$$

Let $u(t)=\widehat{u}(t)-w(t)$, then (3.13) means that $u(t)$ is a positive solution for BVP (1.1)-(1.2).

Next, we rewrite conditions $\left(B_{2}\right)$ and $\left(B_{3}\right)$ as follows:

$\left(B_{2}^{\prime}\right)$ there exists $\widehat{r}_{i}>C\|L\|_{1}(i=1,2, \cdots, m)$ such that

$$
\int_{0}^{1} J(s) \varphi\left(s, \widehat{r}_{i}\right) d s<\widehat{r}_{i}
$$

where $\varphi\left(t, \widehat{r}_{i}\right)=\max \left\{f(t, u):\left(\widehat{r}_{i}-C\|L\|_{1}\right) e(t) \leqslant u \leqslant \widehat{r}_{i}\right\}+L(t) ;$

$\left(B_{3}^{\prime}\right)$ there exists $\widehat{R}_{i}(i=1,2, \cdots, m)$ with

$$
0<\widehat{r}_{1}<\widehat{R}_{1}<\widehat{r}_{2}<\widehat{R}_{2}<\cdots<\widehat{r}_{m}<\widehat{R}_{m}
$$

such that

$$
\int_{0}^{1} \frac{1}{(\alpha-1)} J(s) \psi\left(s, \widehat{R}_{i}\right) d s>\widehat{R}_{i}
$$

where $\psi\left(t, \widehat{R}_{i}\right)=\min \left\{f(t, u):\left(\widehat{R}_{i}-C\|L\|_{1}\right) e(t) \leqslant u \leqslant \widehat{R}_{i}\right\}+L(t)$.

The following theorem on multiple positive solutions for BVP (1.1)-(1.2) can be given easily by Lemma 2.9 .

Theorem 3.3. Suppose that conditions $\left(\mathrm{H}_{1}\right),\left(\mathrm{B}_{1}\right),\left(\mathrm{B}_{2}^{\prime}\right)$, and $\left(\mathrm{B}_{3}^{\prime}\right)$ hold. Then BVP (1.1)-(1.2) has at least $\mathrm{m}$ positive solutions.

Remark 3.4. Similar multiplicity result can be given for the first case, we omit it here.

\section{Examples}

Example 4.1. Consider the following singular fractional differential equation

$$
\left\{\begin{array}{l}
D_{0+}^{\frac{17}{6}} u(t)+\frac{1}{8 \sqrt{t(1-t)}}\left(u^{\frac{1}{2}}+u^{6}\right)-\frac{1}{20 \sqrt[3]{t^{2}}}=0,0<t<1, \\
u(0)=u^{\prime}(0)=0, D_{0+}^{\frac{13}{12}} u(1)=\frac{2}{3} \int_{0}^{\frac{4}{5}} t^{-\frac{3}{4}} D_{0+}^{\frac{13}{12}} u(t) d t .
\end{array}\right.
$$

It is clear, (4.1) has the form of (1.1), where $\alpha=\frac{17}{6}, \beta=\frac{13}{12}, n=3, \lambda=\frac{2}{3}, \eta=\frac{4}{5}, h(t)=t^{-\frac{3}{4}}$. Obviously, $\left(\mathrm{H}_{0}\right)$ holds for $\mathrm{L}(\mathrm{t})=\frac{1}{20 \sqrt[3]{\mathrm{t}^{2}}}$. By simple computation, we know that $\Gamma(\alpha)=1.7245, \alpha-\beta-1=\frac{3}{4}>$ $0, \int_{0}^{\eta} h(t) t^{\alpha-\beta-1} d t=0.8000, \lambda \int_{0}^{\eta} h(t) t^{\alpha-\beta-1} d t=0.5333<1,\|L\|_{1}=0.15, C=3.1639$. It is clear that $\left(A_{1}\right)$ is valid for $\gamma_{\mathrm{r}}(\mathrm{t})=\frac{1}{8 \sqrt{\mathrm{t}(1-\mathrm{t})}}\left(\mathrm{r}^{\frac{1}{2}}+\mathrm{r}^{6}\right)+\frac{1}{20 \sqrt[3]{\mathrm{t}^{2}}}$. 
Next, we check $\left(A_{2}\right)$ and $\left(A_{3}\right)$. It is clear that

$$
\int_{0}^{\eta} h(t) H(t, s) d t<\int_{0}^{\frac{4}{5}} t^{-\frac{3}{4}} \frac{t^{\alpha-\beta-1}}{\Gamma(\alpha)} d t(1-s)^{\alpha-\beta-1}=0.4639(1-s)^{\frac{3}{4}} .
$$

Take $r_{1}=1>0.4746=C\|L\|_{1}$, then we have by (4.2)

$$
\begin{aligned}
\int_{0}^{1} J(s) \varphi(s, 1) \mathrm{d} s & =\int_{0}^{1}\left[\frac{1}{\Gamma(\alpha)}(\alpha-1) s(1-s)^{\alpha-\beta-1}+\frac{\lambda}{1-\lambda \int_{0}^{\eta} h(t) t^{\alpha-\beta-1} \mathrm{dt}} \int_{0}^{\eta} h(t) H(t, s) d t\right] \varphi(s, 1) d s \\
& <\int_{0}^{1}\left[1.0631 s(1-s)^{\frac{3}{4}}+1.4285 \times 0.4639(1-s)^{\frac{3}{4}}\right] \frac{1}{8 \sqrt{s(1-s)}}(1+1) \mathrm{d} s \\
& =\frac{1}{4} \int_{0}^{1}\left[1.0631 s^{\frac{1}{2}}(1-s)^{\frac{1}{4}}+0.6667 s^{-\frac{1}{2}}(1-s)^{\frac{1}{4}}\right] \mathrm{d} s=0.4241<1 .
\end{aligned}
$$

Thus, $\left(A_{2}\right)$ is verified. We have that

$$
\begin{aligned}
\int_{0}^{\eta} h(t) H(t, s) d t & =\int_{0}^{\frac{4}{5}} t^{-\frac{3}{4}} \frac{t^{\alpha-\beta-1}(1-s)^{\alpha-\beta-1}}{\Gamma(\alpha)} d t-\int_{s}^{\frac{4}{5}} t^{-\frac{3}{4}} \frac{(t-s)^{\alpha-\beta-1}}{\Gamma(\alpha)} d t \\
& >\frac{1}{\Gamma(\alpha)} \int_{0}^{\frac{4}{5}} 1 d t(1-s)^{\frac{3}{4}}-\frac{1}{\Gamma(\alpha)} \int_{s}^{\frac{4}{5}} 1 d t=0.4639(1-s)^{\frac{3}{4}}+0.5799 s-0.4639 .
\end{aligned}
$$

Take $a=\frac{2}{3}, b=1, r_{2}=20$, then, we have

$$
\begin{aligned}
\frac{1}{\alpha-1} \int_{a}^{b} J(s) \psi(s, 20) \mathrm{d} s= & \frac{4}{7} \int_{\frac{2}{3}}^{1}\left[\frac{1}{\Gamma(\alpha)}(\alpha-1) s(1-s)^{\alpha-\beta-1}\right. \\
& \left.+\frac{\lambda}{1-\lambda \int_{0}^{\eta} h(t) t^{\alpha-\beta-1} \mathrm{dt}} \int_{0}^{\eta} \mathrm{h}(\mathrm{t}) \mathrm{H}(\mathrm{t}, \mathrm{s}) \mathrm{dt}\right] \psi(\mathrm{s}, 10) \mathrm{d} \mathrm{s} \\
\geqslant & \frac{4}{7} \int_{\frac{2}{3}}^{1}\left[1.0631 \mathrm{~s}(1-\mathrm{s})^{\frac{3}{4}}+1.4285\left(0.4639(1-\mathrm{s})^{\frac{3}{4}}+0.5799 \mathrm{~s}-0.4639\right)\right] \\
& \times \frac{1}{8 \sqrt{s(1-s)}} \min \left\{\left(\mathrm{u}^{\frac{1}{2}}+\mathrm{u}^{6}\right): 5.0649 \leqslant \mathrm{u} \leqslant 20\right\} \mathrm{ds} \\
> & \frac{1}{8} \times \frac{4}{7} \times 5.0649^{6} \int_{\frac{2}{3}}^{1}\left[1.0631 \mathrm{~s}^{\frac{1}{2}}(1-\mathrm{s})^{\frac{1}{4}}+1.4285\left(0.4639 \mathrm{~s}^{-\frac{1}{2}}(1-\mathrm{s})^{\frac{1}{4}}\right.\right. \\
& \left.\left.+0.5799 \mathrm{~s}^{\frac{1}{2}}(1-\mathrm{s})^{-\frac{1}{2}}-0.4639 \mathrm{~s}^{-\frac{1}{2}}(1-\mathrm{s})^{-\frac{1}{2}}\right)\right] \mathrm{ds} \\
> & \frac{1}{8} \times \frac{4}{7} \times 5.0649^{6} \int_{\frac{2}{3}}^{1}\left[1.0631\left(\frac{2}{3}\right)^{\frac{1}{2}}(1-\mathrm{s})^{\frac{1}{4}}+1.4285\left(0.4639(1-\mathrm{s})^{\frac{1}{4}}\right.\right. \\
& \left.\left.+0.5799\left(\frac{2}{3}\right)^{\frac{1}{2}}(1-\mathrm{s})^{-\frac{1}{2}}-0.4639 \mathrm{~s}^{-\frac{1}{2}}(1-\mathrm{s})^{-\frac{1}{2}}\right)\right] \mathrm{ds} \\
= & \frac{1}{8} \times \frac{4}{7} \times 16882 \times[1.0631 \times 0.1654+1.4285(0.4639 \times 0.2026 \\
& +0.5799 \times 0.9428-0.4639 \times 1.2310)] \\
= & 87.7864>20 .
\end{aligned}
$$

Thus, $\left(\mathrm{A}_{3}\right)$ is verified. It follows from Theorem 3.1 that BVP (4.1) has at least one positive solution.

Example 4.2. Consider the following singular fractional differential equation

$$
\left\{\begin{array}{l}
D_{0+}^{\frac{5}{2}} u(t)+\frac{1}{48 \sqrt[4]{t^{2}(1-t)}}\left(u^{5}+\frac{1}{\sqrt[6]{u}}\right)-\frac{1}{15 \sqrt[5]{t^{2}}}=0,0<t<1, \\
u(0)=u^{\prime}(0)=0, D_{0+}^{\frac{5}{4}} u(1)=\frac{1}{2} \int_{0}^{\frac{2}{3}} t^{-\frac{1}{6}} D_{0+}^{\frac{5}{4}} u(t) d t .
\end{array}\right.
$$


It is clear, (4.3) has the form of (1.1), where $\alpha=\frac{5}{2}, \beta=\frac{5}{4}, \mathrm{n}=3, \lambda=\frac{1}{2}, \eta=\frac{2}{3}, \mathrm{~h}(\mathrm{t})=\mathrm{t}^{-\frac{1}{6}}$. Obviously, $\left(\mathrm{H}_{1}\right)$ holds for $\mathrm{L}(\mathrm{t})=\frac{1}{15 \sqrt[5]{\mathrm{t}^{2}}}$. By simple computation, we know that $\Gamma(\alpha)=1.3293, \int_{0}^{\eta} \mathrm{h}(\mathrm{t}) \mathrm{t}^{\alpha-\beta-1} \mathrm{dt}=$ $0.5949, \lambda \int_{0}^{\eta} h(t) t^{\alpha-\beta-1} d t=0.2974<1, C=2.1702,\|L\|_{1}=\frac{1}{9}$. It is clear that $\left(B_{1}\right)$ is valid for $\gamma_{r_{1}, r_{2}}=$ $\frac{1}{48 \sqrt[4]{t^{2}(1-t)}}\left[r_{2}{ }^{5}+\left(\frac{2}{3} r_{1} t^{\frac{3}{2}}\right)^{-\frac{1}{6}}\right]+\frac{1}{15 \sqrt[5]{t^{2}}}$. Next, we check $\left(B_{2}\right)$ and $\left(B_{3}\right)$. It is clear that

$$
\int_{0}^{\eta} h(t) H(t, s) d t<\int_{0}^{\frac{2}{3}} t^{-\frac{1}{6}} \frac{t^{\alpha-\beta-1}}{\Gamma(\alpha)} d t(1-s)^{\alpha-\beta-1}=0.4475(1-s)^{\frac{1}{4}}
$$

Take $r_{1}=2>0.2411=C\|\mathrm{~L}\|_{1}$, then by (4.3) we have

$$
\begin{aligned}
\int_{0}^{1} J(s) & \varphi(s, 2) \mathrm{d} s \\
= & \int_{0}^{1}\left[\frac{1}{\Gamma(\alpha)}(\alpha-1) s(1-s)^{\frac{1}{4}}\right. \\
& \left.+\frac{\lambda}{1-\lambda \int_{0}^{\eta} h(t) t^{\alpha-\beta-1} \mathrm{dt}} \int_{0}^{\eta} h(t) H(t, s) \mathrm{d} t\right] \frac{1}{48 \sqrt[4]{s^{2}(1-s)}} \max \left\{\left(u^{5}+\frac{1}{\sqrt[6]{u}}\right): 1.1726 s^{\frac{3}{2}} \leqslant u \leqslant 2\right\} \mathrm{d} s \\
< & \frac{1}{48} \int_{0}^{1}\left[1.1284 s^{\frac{1}{2}}+0.7116 \times 0.4475 s^{-\frac{1}{2}}\right]\left(2^{5}+\frac{1}{\sqrt[6]{1.1726 s^{\frac{3}{2}}}}\right) \mathrm{d} s \\
= & \frac{1}{48} \times 2^{5}(1.1284 \times 0.6667+0.7116 \times 0.4475 \times 2) \\
& +\frac{1}{48} \times 0.9738 \times \int_{0}^{1}\left[1.1284 s^{\frac{1}{4}}+0.7116 \times 0.4475 s^{-\frac{3}{4}}\right] \mathrm{d} s \\
= & 0.9261+0.0442=0.9703<2 .
\end{aligned}
$$

Thus, $\left(B_{2}\right)$ is verified. We have that

$$
\begin{aligned}
\int_{0}^{\eta} h(t) H(t, s) d t & =\int_{0}^{\frac{2}{3}} t^{-\frac{1}{6}} \frac{t^{\alpha-\beta-1}(1-s)^{\alpha-\beta-1}}{\Gamma(\alpha)} d t-\int_{s}^{\frac{2}{3}} t^{-\frac{1}{6}} \frac{(t-s)^{\alpha-\beta-1}}{\Gamma(\alpha)} d t \\
& >\frac{1}{\Gamma(\alpha)} \int_{0}^{\frac{2}{3}} t^{\frac{1}{12}} d t(1-s)^{\frac{1}{4}}-\frac{1}{\Gamma(\alpha)} \int_{s}^{\frac{2}{3}} t^{\frac{1}{12}} d t \\
& =0.4475(1-s)^{\frac{1}{4}}+0.6944 s^{\frac{13}{12}}-0.4475 .
\end{aligned}
$$

Take $r_{2}=15$, then, we have

$$
\begin{aligned}
& \frac{1}{\alpha-1} \int_{0}^{1} J(s) \varphi(s, 15) \mathrm{d} s \\
&=\frac{1}{\alpha-1} \int_{0}^{1}\left[\frac{1}{\Gamma(\alpha)}(\alpha-1) s(1-s)^{\frac{1}{4}}\right. \\
&\left.\quad+\frac{\lambda}{1-\lambda \int_{0}^{\eta} h(t) t^{\alpha-\beta-1} \mathrm{~d} t} \int_{0}^{\eta} h(t) H(t, s) d t\right] \frac{1}{48 \sqrt[4]{s^{2}(1-s)}} \min \left\{\left(u^{5}+\frac{1}{\sqrt[6]{u}}\right): 9.8393 s^{\frac{3}{2}} \leqslant u \leqslant 15\right\} d s \\
&> \frac{2}{3} \times \frac{1}{48} \int_{0}^{1}\left[1.1284 s^{\frac{1}{2}}+0.7116 \times 0.4475 s^{-\frac{1}{2}}+0.7116 \times 0.6944 s^{\frac{7}{12}}(1-s)^{-\frac{1}{4}}\right. \\
&\left.-0.7116 \times 0.4475 s^{-\frac{1}{2}}(1-s)^{-\frac{1}{4}}\right]\left(9.8393^{5} s^{\frac{15}{2}}+\frac{1}{\sqrt[6]{15}}\right) d s \\
&> \frac{2}{3} \times \frac{1}{48} \times 9.8393^{5} \int_{0}^{1}\left[1.1284 s^{8}+0.7116 \times 0.4475 s^{7}\right.
\end{aligned}
$$




$$
\begin{aligned}
& \left.+0.7116 \times 0.6944 s^{\frac{97}{12}}(1-s)^{-\frac{1}{4}}-0.7116 \times 0.4475 s^{7}(1-s)^{-\frac{1}{4}}\right] \mathrm{ds} \\
= & \frac{1}{72} \times 92219\left(1.1284 \times \frac{1}{9}+0.7116 \times 0.4475 \times \frac{1}{8}+0.7116 \times 0.6944 \times 0.2366\right. \\
& -0.7116 \times 0.4475 \times 0.2606) \\
= & 1281.8 \times 0.1991=255.2064>15 .
\end{aligned}
$$

Thus, $\left(B_{3}\right)$ is verified. It follows from Theorem 3.2 that BVP (4.3) has at least one positive solution.

\section{Acknowledgment}

The project is supported financially by the Foundations for NSFC cultivation project of Jining Medical University, a Project of Shandong Province Higher Educational Science and Technology Program (J15LI16), the Natural Science Foundation of Jining Medical University (JY2015BS07, JY2015KJ019), the Natural Science Foundation of Shandong Province of China (ZR2015AL002), and the National Natural Science Foundation of China $(11571296,11571197,11371221,11071141)$.

\section{References}

[1] R. P. Agarwal, D. O'Regan, Positive solutions for $(\mathrm{p}, \mathrm{n}-\mathrm{p})$ conjugate boundary value problems, J. Differential Equations, 150 (1998), 462-473. 1, 1

[2] R. P. Agarwal, D. O'Regan, Twin solutions to singular boundary value problems, Proc. Amer. Math. Soc., 128 (2000), 2085-2094.

[3] R. P. Agarwal, D. O’Regan, Multiplicity results for singular conjugate, focal, and ( $n, p)$ problems, J. Differential Equations, 170 (2001), 142-156. 1, 1

[4] B. Ahmad, S. K. Ntouyas, A fully Hadamard type integral boundary value problem of a coupled system of fractional differential equations, Fract. Calc. Appl. Anal., 17 (2014), 348-360. 1

[5] Z.-B. Bai, On positive solutions of a nonlocal fractional boundary value problem, Nonlinear Anal., 72 (2010), $916-924$. 2.3, 2.4

[6] D. Baleanu, K. Diethelm, E. Scalas, J. J. Trujillo, Fractional calculus. Models and numerical methods, Series on Complexity, Nonlinearity and Chaos, World Scientific Publishing Co. Pte. Ltd., Hackensack, NJ, (2012). 1

[7] A. Cabada, Z. Hamdi, Nonlinear fractional differential equations with integral boundary value conditions, Appl. Math. Comput., 228 (2014), 251-257. 1

[8] A. Cabada, G.-T. Wang, Positive solutions of nonlinear fractional differential equations with integral boundary value conditions, J. Math. Anal. Appl., 389 (2012), 403-411. 1, 1, 1, 1

[9] Y.-J. Cui, Y.-M. Zou, Monotone iterative technique for $(\mathrm{k}, \mathrm{n}-\mathrm{k})$ conjugate boundary value problems, Electron. J. Qual. Theory Differ. Equ., 2015 (2015), 11 pages. 1

[10] P. W. Eloe, J. Henderson, Singular nonlinear boundary value problems for higher order ordinary differential equations, Nonlinear Anal., 17 (1991), 1-10.

[11] P. W. Eloe, J. Henderson, Existence of solutions for some singular higher order boundary value problems, Z. Angew. Math. Mech., 73 (1993), 315-323.

[12] P. W. Eloe, J. Henderson, Positive solutions for $(n-1,1)$ conjugate boundary value problems, Nonlinear Anal., 28 (1997), 1669-1680.

[13] P. W. Eloe, J. Henderson, Singular nonlinear $(\mathrm{k}, \mathrm{n}-\mathrm{k})$ conjugate boundary value problems, J. Differential Equations, 133 (1997), 136-151. 1

[14] M.-Q. Feng, Existence of symmetric positive solutions for a boundary value problem with integral boundary conditions, Appl. Math. Lett., 24 (2011), 1419-1427. 1

[15] J. R. Graef, T. Moussaoui, A class of nth-order BVPs with nonlocal conditions, Comput. Math. Appl., 58 (2009), 1662-1671. 1

[16] D. J. Guo, V. Lakshmikantham, Nonlinear problems in abstract cones, Notes and Reports in Mathematics in Science and Engineering, Academic Press, Inc., Boston, MA, (1988). 2.9

[17] L.-M. Guo, C. Liu, Existence of positive solutions for singular higher-order fractional differential equation via spectral analysis, J. Appl. Math. Comput., 54 (2017), 357-379. 1

[18] L.-M. Guo, L.-S. Liu, Y.-H. Wu, Existence of positive solutions for singular fractional differential equations with infinitepoint boundary conditions, Nonlinear Anal. Model. Control, 21 (2016), 635-650.

[19] L.-M. Guo, L.-S. Liu, Y.-H. Wu, Existence of positive solutions for singular higher-order fractional differential equations with infinite-point boundary conditions, Bound. Value Probl., 2016 (2016), 22 pages.

[20] X.-N. Hao, L.-S. Liu, Y.-H. Wu, Q. Sun, Positive solutions for nonlinear nth-order singular eigenvalue problem with nonlocal conditions, Nonlinear Anal., 73 (2010), 1653-1662. 1 
[21] J. Henderson, R. Luca, Positive solutions for a system of nonlocal fractional boundary value problems, Fract. Calc. Appl. Anal., 16 (2013), 985-1008.

[22] J. Henderson, R. Luca, Positive solutions for a system of fractional differential equations with coupled integral boundary conditions, Appl. Math. Comput., 249 (2014), 182-197.

[23] G. Infante, P. Pietramala, M. Tenuta, Existence and localization of positive solutions for a nonlocal BVP arising in chemical reactor theory, Commun. Nonlinear Sci. Numer. Simul., 19 (2014), 2245-2251. 1

[24] D.-Q. Jiang, J.-F. Chu, M.-R. Zhang, Multiplicity of positive periodic solutions to superlinear repulsive singular equations, J. Differential Equations, 211 (2005), 282-302. 1

[25] A. A. Kilbas, H. M. Srivastava, J. J. Trujillo, Theory and applications of fractional differential equations, North-Holland Mathematics Studies, Elsevier Science B.V., Amsterdam, (2006). 1, 2.1, 2.2

[26] L.-J. Kong, Q.-K. Kong, Higher order boundary value problems with nonhomogeneous boundary conditions, Nonlinear Anal., 72 (2010), 240-261. 1

[27] L.-B. Kong, J.-Y. Wang, The Green's function for $(\mathrm{k}, \mathrm{n}-\mathrm{k})$ conjugate boundary value problems and its applications, J. Math. Anal. Appl., 255 (2001), 404-422. 1, 1

[28] L.-S. Liu, X.-N. Hao, Y.-H. Wu, Positive solutions for singular second order differential equations with integral boundary conditions, Math. Comput. Modelling, 57 (2013), 836-847. 1

[29] I. Podlubny, Fractional differential equations, An introduction to fractional derivatives, fractional differential equations, to methods of their solution and some of their applications, Mathematics in Science and Engineering, Academic Press, Inc., San Diego, CA, (1999). 1

[30] J. Sabatier, O. P. Agrawal, J. A. Tenreiro Machado (eds.), Advances in fractional calculus, Theoretical developments and applications in physics and engineering, Including papers from the Minisymposium on Fractional Derivatives and their Applications (ENOC-2005) held in Eindhoven, August 2005, and the 2nd Symposium on Fractional Derivatives and their Applications (ASME-DETC 2005) held in Long Beach, CA, September 2005, Springer, Dordrecht, (2007). 1

[31] S. G. Samko, A. A. Kilbas, O. I. Marichev, Fractional integrals and derivatives, Theory and applications, Edited and with a foreword by S. M. Nikol'ski1̌, Translated from the 1987 Russian original, Revised by the authors, Gordon and Breach Science Publishers, Yverdon, (1993). 1

[32] Q. Sun, Y.-M. Cui, Solvability of $(\mathrm{k}, \mathrm{n}-\mathrm{k})$ conjugate boundary value problems with integral boundary conditions at resonance, J. Funct. Spaces, 2016 (2016), 7 pages. 1

[33] Y.-Q. Wang, L.-S. Liu, Y.-H. Wu, Positive solutions for a nonlocal fractional differential equation, Nonlinear Anal., 74 (2011), 3599-3605. 1, 1

[34] L. Wang, X.-Q. Zhang, Existence of positive solutions for a class of higher-order nonlinear fractional differential equations with integral boundary conditions and a parameter, J. Appl. Math. Comput., 44 (2014), 293-316. 1, 1

[35] J. R. L. Webb, Nonlocal conjugate type boundary value problems of higher order, Nonlinear Anal., 71 (2009), $1933-1940$. 1

[36] Q.-L. Yao, Local existence of multiple positive solutions to a singular cantilever beam equation, J. Math. Anal. Appl., 363 (2010), 138-154. 1

[37] Q.-L. Yao, Positive solutions of nonlinear beam equations with time and space singularities, J. Math. Anal. Appl., 374 (2011), 681-692. 1

[38] X.-Q. Zhang, Positive solutions for a class of singular fractional differential equation with infinite-point boundary value conditions, Appl. Math. Lett., 39 (2015), 22-27. 1, 1

[39] X.-Q. Zhang, Positive solutions for singular higher-order fractional differential equations with nonlocal conditions, J. Appl. Math. Comput., 49 (2015), 69-89. 1, 1

[40] L.-H. Zhang, B. Ahmad, G.-T. Wang, The existence of an extremal solution to a nonlinear system with the right-handed Riemann-Liouville fractional derivative, Appl. Math. Lett., 31 (2014), 1-6.

[41] X.-U. Zhang, Y.-F. Han, Existence and uniqueness of positive solutions for higher order nonlocal fractional differential equations, Appl. Math. Lett., 25 (2012), 555-560.

[42] X.-U. Zhang, L.-S. Liu, Y.-H. Wu, The uniqueness of positive solution for a fractional order model of turbulent flow in a porous medium, Appl. Math. Lett., 37 (2014), 26-33.

[43] X.-U. Zhang, L.-S. Liu, Y.-H. Wu, B. Wiwatanapataphee, The spectral analysis for a singular fractional differential equation with a signed measure, Appl. Math. Comput., 257 (2015), 252-263.

[44] X.-Q. Zhang, L. Wang, Q. Sun, Existence of positive solutions for a class of nonlinear fractional differential equations with integral boundary conditions and a parameter, Appl. Math. Comput., 226 (2014), 708-718. 1, 1, 1

[45] Y. Zhou, Basic theory of fractional differential equations, World Scientific Publishing Co. Pte. Ltd., Hackensack, NJ, (2014). 1 